\title{
The Toroidal Field Coil Design for ARIES-ST
}

\author{
W. Reiersen, F. Dahlgren, H-M. Fan, C. Neumeyer, and I. Zatz \\ Princeton Plasma Physics Laboratory, Princeton, NJ 08543
}

Abstract- An evolutionary process was used to develop the toroidal field (TF) coil design for the ARIES-ST (Spherical Tokamak). Design considerations included fabricability, assembly, maintenance, energy efficiency, and structural robustness. The design addresses a number of the concerns (complexity) and criticisms (high cost, high recirculating power) of fusion. It does this by:

- Applying advanced, but available laser forming and spray casting techniques for manufacturing the $\mathrm{TF}$ coil system;

- Adopting a simple single toroidal field coil system to make assembly and maintenance much easier. The single turn design avoids the necessity of using the insulation as a structural component of the TF coils, and hence is much more robust than multi-turn designs.

- Using a high conductivity copper alloy and modest current densities to keep the recirculating power modest.

\section{INTRODUCTION}

The goal of this study was to develop an attractive TF coil configuration for a spherical tokamak (ST) reactor. The TF system must be capable of providing a field of $2.14 \mathrm{~T}$ at a major radius of $3.2 \mathrm{~m}$ and be compatible with the overall maintenance concepts.

\section{TF COIL SYSTEM DESIGN}

The configuration concept for the single turn coil system adopted for ARIES-ST is illustrated in Figure 1. A single turn centerpost (CP) configuration can carry more current than a multi-turn configuration of the same radius because of its higher packing fraction (no insulation, flexible

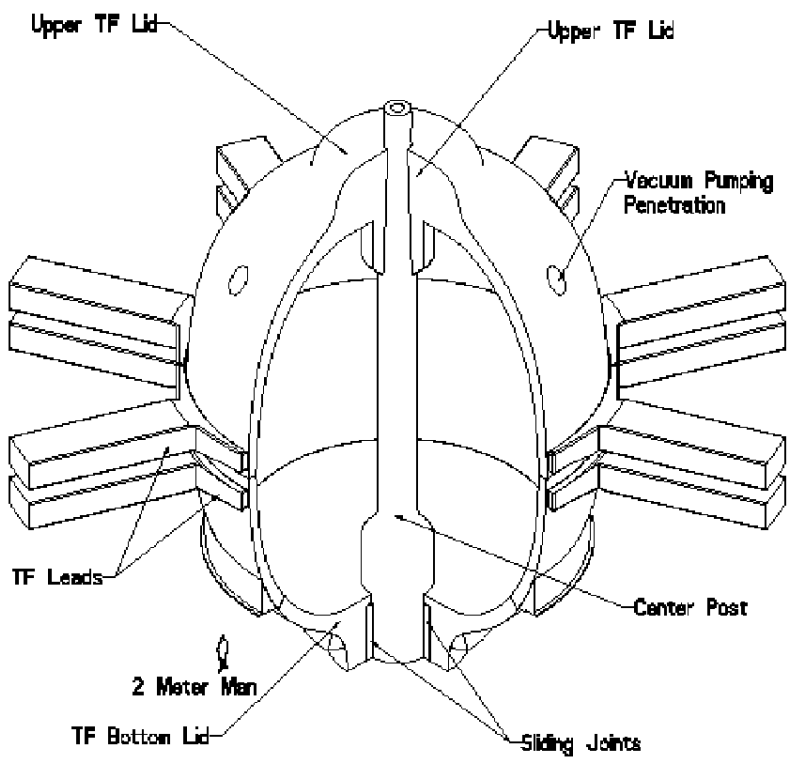

Figure 1 - Isometric view of TF coil

conductor geometry) and reduced shielding requirement (no insulation). The large conductor currents in the single turn configuration, while formidable, do not appear intractable from a power supply standpoint. A scheme for providing a very high current (multi-mega-ampere) power supply has been developed. The scheme uses a large number of diode rectifiers connected in parallel to provide current to the TF load

The configuration features a tall $\mathrm{CP}$ that is oriented along the major axis of the machine. The $\mathrm{CP}$ is connected to an outer shell that surrounds the first wall, blanket, shield, divertors, and PF coils. The TF system provides the primary vacuum boundary for the machine.

The $\mathrm{CP}$ is designed to be physically separable from the power core assembly. The bottom portion of the $\mathrm{CP}$ is a thick cylinder. It is electrically connected to the outer shell by sliding joints. The CP and outer shell are keyed together in this location, permitting relative motion radially and vertically while keeping them registered toroidally. Numerous concepts for sliding electrical contacts have been developed, tested, and even deployed for fusion applications. These concepts include; Feltmetal pads (used on C-Mod and proposed on MAST), Multilam sliding contacts [1], and spring-loaded, in-line contacts [2]. Additionally, liquid metal joints have been considered. For this study, a sliding joint utilizing Feltmetal pads was assumed in developing the configuration concept. In addition to easing assembly and maintenance, the sliding joints significantly reduces axial stresses in the $\mathrm{CP}$.

The $\mathrm{CP}$ transitions from a large diameter $(3.2 \mathrm{~m})$ cylinder at the bottom to a smaller diameter $(1.8 \mathrm{~m})$ cylinder between the upper and lower divertors. This is the region of high current density, accounting for most of the Joule losses. Flaring the $\mathrm{CP}$ on top as it is on the bottom would have trapped the $\mathrm{CP}$ with the power core assembly. Instead, the flaring is incorporated into the upper section of the outer shell. The CP has a conical shape above the upper divertor assembly where it is pulled against a mating surface in the outer shell for electrical continuity. Gravity support of the $\mathrm{CP}$ and the preload for the required contact pressure between the upper part of the $\mathrm{CP}$ and outer shell are provided where the CP penetrates the top of the outer shell. This arrangement allows the $\mathrm{CP}$ to be removed either without disturbing the power core assembly or as part of the power core assembly, as shown in Figure 2. It also minimizes Joule losses by restricting the region of high current to the cylindrical section between the upper and lower divertors. Since the TF provides the primary vacuum boundary, there are bellows connections above and below the outer shell where the $\mathrm{CP}$ penetrates the outer shell to provide vacuum seals.

The outer shell is segmented into three pieces, as shown in Figure 1. The lower section of the outer shell provides the gravity support for the power core assembly, including the first wall, blanket, inboard shield, divertors, and lower PF coils. The lower section is in turn supported by removable supports from below. The upper section extends from the $\mathrm{CP}$ to the outboard midplane. At the outboard midplane, there is a joint between the upper section and middle section. The upper and middle sections are bolted together with an electrically insulating material in between. A bellows-type connection (with an insulating break) on the inside of the outer shell provides the vacuum barrier across the joint. The TF leads connect to the upper and middle sections at eight equally spaced toroidal locations. 
The middle section of the outer shell is connected to the lower section at approximately the same elevation as the lower divertor. At this elevation, the major radius of the joint is adequate to permit vertical removal of the power core. This joint provides the electrical continuity between the middle and lower sections. For removal of the bottom section of the outer shell and power core, the bellows connection is cut and the joint unbolted. The connections between the $\mathrm{CP}$ and upper section of the outer shell must also be undone. The middle section of the outer shell is supported off the floor of the test cell. These supports bear the gravity loads of the middle and upper sections of the outer shell, the $\mathrm{CP}$, and the upper PF coils attached to the outer shell.

When fully assembled, the outer shell provides a continuous shell that is very effective in reacting both inplane and out-of-plane electromagnetic loads. In additional to the $\mathrm{CP}$, penetrations will be required for helium cooling in the power core, the $\mathrm{Li}-\mathrm{Pb}$ breeding material, vacuum pumping, neutral beam injection, and PF coil services. Some diagnostic penetrations are also likely to be required. Vacuum seals and electrical isolation will have to be provided at all these locations.

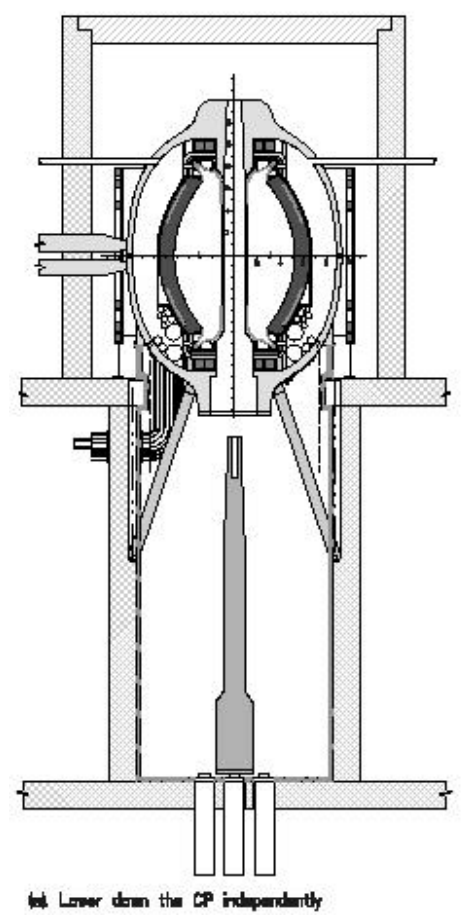

Figure 2 - Elevation view showing fusion power core maintenance paths

\section{MATERIAL SELECTION}

Copper alloys are available that provide higher strength than copper, especially at elevated temperatures, but with reduced electrical and thermal conductivity. For this study, two alloys were considered - the precipitation hardened $(\mathrm{PH})$ alloy $\mathrm{CuCrZr}$ (with a nominal composition of $\mathrm{Cu}-0.65 \% \mathrm{Cr}-0.15 \% \mathrm{Zr}$ ) and the dispersion strengthened (DS) alloy Glidcop AL-15 (with a nominal composition of $\mathrm{Cu}$ $0.15 \mathrm{Al}$ as oxide particles $0.28 \% \mathrm{Al}_{2} \mathrm{O}_{3}$ ). These alloys represent two different classes of materials. Both have been well characterized in a radiation environment as a result of extensive testing conducted on the ITER project.
Both materials exhibit very good strength (greater than $300 \mathrm{MPa}$ TYS and $400 \mathrm{MPa}$ UTS) at room temperature in the unirradiated condition. Because of the complexity of manufacturing the $\mathrm{CP}$, no cold working was assumed for comparing material properties. The electrical conductivity of Glidcop AL-15 is approximately 90\% IACS at room temperature, significantly higher than $\mathrm{CuCrZr}$ with an electrical conductivity of approximately $80 \%$.

Irradiation at temperatures below $150^{\circ} \mathrm{C}$ causes hardening in pure copper and precipitation hardened (PH) and dispersion strengthened (DS) copper alloys. Hardening resulting from low temperature irradiation is accompanied by severe embrittlement [3] in PH and DS alloys. The uniform elongation generally decreases to less than $1 \%$ even at doses as low as 0.01 to $0.1 \mathrm{dpa}$. The expected peak dose at the $\mathrm{CP}$ after 1 full power year (FPY) of operation is 12 dpa. Thus, these materials, if used in the $\mathrm{CP}$ and irradiated at low temperatures (less than $150^{\circ} \mathrm{C}$ ), would be brittle. To avoid the higher Joule losses associated with high conductor temperatures, the inlet temperature for the reference design was set at $30^{\circ} \mathrm{C}$, slightly above ambient temperature. Peak temperatures were below $100^{\circ} \mathrm{C}$. Brittle material allowables were used in evaluating the design. At temperatures less

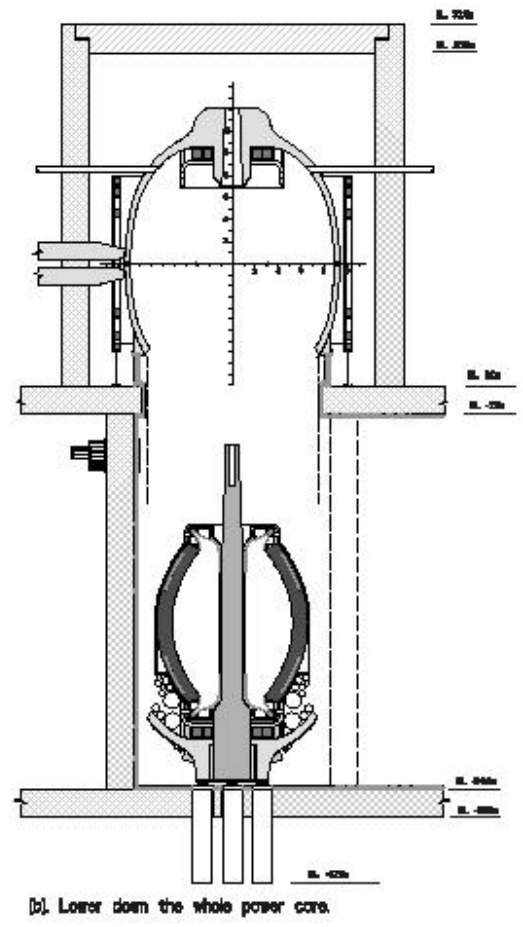

than $150^{\circ} \mathrm{C}$ with stress levels below $250 \mathrm{MPa}$, creep is not expected to be an issue. Likewise, swelling should not be significant at temperatures less than $150^{\circ} \mathrm{C}[3]$.

All copper alloys will be subject to transmutations that decrease the electrical and thermal conductivity and elevate the waste disposal rating (WDR). One of the requirements for ARIES-ST is to limit the WDR of the TF coils to Class C waste. With a $20 \mathrm{~cm}$ helium-cooled, ferritic steel (FS) shield, the design life of the CP in ARIES-ST is 3 FPY using 10CFR61 limits and 6 FPY using Fetter limits. The design life of the ferritic shield structures surrounding the plasma is 3 FPY so the CP would have to be replaced either every replacement or every second replacement of the FS structures, depending on which limits were used, in order to satisfy WDR requirements. The 10CFR61 WDR is determined mainly from the long-lived isotopes produced 
from the $\mathrm{Cu}$ itself (Ni-63), not from the alloying elements. The Fetter WDR is determined mainly from Ag-108m, produced from silver impurities that are not presently controlled in the material specifications. In either case, the WDR does not appear to be differentiating factor in selecting the conductor material.

Calculations were performed to assess the increase in the electrical resistance of the $\mathrm{CP}$ over time due to transmutations. Initially, the current distribution in the $\mathrm{CP}$ is nearly uniform. The decrease near the outer edge is due to the higher temperature resulting from nuclear heating. Over time, the current density near the outer edge drops dramatically due to the local increase in electrical resistivity. The current density in the center of the CP increases to keep the total current constant. The net effect is that the resistance of the CP increases more than $4 \%$ per FPY. After 3 FPY, the CP resistance would increase by approximately $12.5 \%$. This represents a decrease in net electrical power of approximately 33MW. After $6 \mathrm{FPY}$, the $\mathrm{CP}$ resistance would increase by approximately $23 \%$, resulting in a decrease in net electrical power of 58MW. Plant economics appear to favor replacing the CP every 3 FPY (corresponding to the replacement time for the plasma facing FS structures) because the $\mathrm{CP}$ is relatively inexpensive $(\$ 7 \mathrm{M})$, although WDR considerations might allow a 6 FPY replacement time.

The economics of fusion power are driven by the capital cost of the plant. The cost of the magnet systems has traditionally been a large element in the overall capital cost. For ARIES-ST, a study of low cost fabrication options for the TF system was performed by the Boeing Company with support from the AeroMet Corporation. Two low cost fabrication methods were identified - laser forming for the $\mathrm{CP}$ and spray casting for the TF outer shell. The cost of laser forming the CP from powdered copper was estimated to be approximately $\$ 8 / \mathrm{kg}$. The cost of spray casting the outer shell from molten aluminum was approximately $\$ 4 / \mathrm{kg}$. Applying these methods substantially lower the capital cost of the plant.

Use of the laser forming technique for fabricating the $\mathrm{CP}$ may be a differentiating factor in selecting the best copper alloy. For Glidcop AL-15, a copper alloy which is dispersion strengthened with $\mathrm{Al}_{2} \mathrm{O}_{3}$, it would be difficult to use the current technology for laser forming as the $\mathrm{Al}_{2} \mathrm{O}_{3}$ particles would tend to redistribute during the melting process. Using a precipitation hardened alloy such as $\mathrm{CuCrZr}$ may be feasible if the material could be properly heat treated either during or after the deposition and shaping process [4]. $\mathrm{CuCrZr}$ properties were used for this study because it appeared more feasible to fabricate the $\mathrm{CP}$ using laser forming with this material. However, from a broad perspective, these two alloys represent a class of materials (high conductivity, high strength copper alloys, with good fabrication characteristics) that appears able to meet the requirements for the $\mathrm{CP}$ of a low aspect ratio, tokamak reactor.

The outer shell is constructed of a 5000 series aluminum alloy. This class of conductor was chosen because the outer shell can be fabricated using a spray casting technique. There are alloys in this series that exhibit good electrical conductivity $(\sim 3.7 \mu \Omega-\mathrm{cm})$ and weldability. The outer shell thickness is approximately $0.7 \mathrm{~m}$. This thickness results in low stresses, low current density in the outer shell $\left(0.07 \mathrm{kA} / \mathrm{cm}^{2}\right)$, and low Joule losses (31 MW).

\section{ELECTRICAL CHARACTERISTICS}

Electrically, the TF system has only a single turn. 34.3 MA are required to satisfy the toroidal field requirement of $2.14 \mathrm{~T}$ at $3.2 \mathrm{~m}$. The voltage drop across the TF coil system is $8.5 \mathrm{~V}$, corresponding to Joule losses of $291 \mathrm{MW}$. The Joule losses occur predominantly in the CP (242 MW) with the balance occurring in the outer shell (31 MW) and bus (19 $\mathrm{MW})$. The current density in the $\mathrm{CP}$ conductor at the midplane is $1.47 \mathrm{kA} / \mathrm{cm}^{2}$. The electrical resistivity of $\mathrm{CuCrZr}$ is $80 \%$ IACS at room temperature. The stored energy in the TF coil system is $6.2 \mathrm{GJ}$.

\section{THERMAL-HYDRAULIC PERFORMANCE}

The $\mathrm{CP}$ is cooled with water with a $30^{\circ} \mathrm{C}$ inlet temperature, slightly above ambient temperature. The water is fed in at the top and exits at the bottom. The feed on top was chosen to minimize thermal stresses across the contact interface between the $\mathrm{CP}$ and outer shell. The top plenum feeds 386 individual circular cooling passages that are collected in a plenum at the bottom. Each cooling passage has a diameter of approximately $2.6 \mathrm{~cm}$ with an average spacing of $9.2 \mathrm{~cm}$. The coolant holes are more closely spaced towards the plasma because that is where the nuclear heating is localized. The coolant fraction is approximately $8.3 \%$ at the midplane of the CP.

The heat load to be removed from the CP during initial operation is approximately $406 \mathrm{MW}$, consisting of $242 \mathrm{MW}$ of Joule heating and $164 \mathrm{MW}$ of nuclear heating. The coolant velocity was limited to $10 \mathrm{~m} / \mathrm{s}$ to avoid excessive erosion. The required flow rate is $2155 \mathrm{~kg} / \mathrm{s}$ with an outlet temperature of $75^{\circ} \mathrm{C}$. To achieve this flow rate, the required inlet pressure is a modest $1.36 \mathrm{MPa}$ with an outlet pressure of $0.15 \mathrm{MPa}$. The average conductor temperature at the midplane is approximately $33^{\circ} \mathrm{C}$ higher than the bulk temperature of the coolant due to an $18^{\circ} \mathrm{C}$ film temperature rise and $15^{\circ} \mathrm{C}$ temperature rise across the conductor. The Joule heating in the CP increases by 33MW after 3 FPY. With the same flow rate, the outlet temperature increases by $4^{\circ} \mathrm{C}$ to $79^{\circ} \mathrm{C}$. Provisions for auxiliary cooling of the $\mathrm{CP}$ for removal of the modest afterheat (approximately $2 \mathrm{~kW}$ maximum) should be made.

The heat load to be removed from the outer shell is low $(70 \mathrm{MW})$. Nuclear heat loads in the outer shell are approximately $21 \mathrm{MW}$ in the lightly shielded collar and only $18 \mathrm{MW}$ elsewhere. The $70 \mathrm{MW}$ total heat load is removed by water flowing through stainless steel tubes embedded in the spray cast outer shell.

\section{STRUCTURAL ANALYSIS}

A preliminary structural analysis of the ARIES-ST TF coil system was performed. The $\mathrm{CP}$ is subject to radial, compressive loads due to the vertical TF current crossing the toroidal field. These radial loads result in stresses in the toroidal direction, i.e. hoop stresses, that range from $16 \mathrm{MPa}$ (compression) at the outer surface to $60 \mathrm{MPa}$ (compression) in the center. In addition, there is a vertical separating force on the $\mathrm{CP}$ due to radial currents near the ends of the $\mathrm{CP}$ crossing the toroidal field. Because the diameter of the $\mathrm{CP}$ is larger at the bottom than at the top, there is also a net downward vertical force on the CP. The net vertical load is reacted where the $\mathrm{CP}$ is attached to the outer shell at the top. Sufficient preload must be provided at this attachment to maintain adequate contact pressure across the joint, which provides electrical continuity between the $\mathrm{CP}$ and outer shell.

The CP is free to expand vertically. Vertical forces on the $\mathrm{CP}$ result in axial tensile stresses ranging from $24 \mathrm{MPa}$ (tension) in the center to $46 \mathrm{MPa}$ (tension) at the outer surface of the CP. The axial stresses are smaller in magnitude and opposite in sign to the hoop stresses. They peak on the outer surface whereas the hoop stresses peak in the center. The VonMises stress in the CP is fairly uniform along its length, ranging from $55 \mathrm{MPa}$ at the outer surface to $76 \mathrm{MPa}$ in the center.

Stresses in the outer shell are low everywhere, with a VonMises stress less than $21 \mathrm{MPa}$ except in a very local 
region near the corner by the collar where the stresses are less than $30 \mathrm{MPa}$. The continuous outer shell is ideal for resisting both in-plane and out-of-plane loads. The absence of turn-to-turn insulation in a single turn coil design removes the traditional weak link for stresses arising from out-ofplane loads. The only electrical insulation is at the outboard midplane. Interlaminar shear stresses are very low in this region, less than $4 \mathrm{MPa}$. More detailed design and analysis are required to quantify stresses due to out-of-plane loads where the $\mathrm{CP}$ interfaces with the outer shell at the top and bottom.

For calculating allowable stresses in the ARIES-ST design, the ITER criteria [5] were followed. In general, for structural materials, the design Tresca stress value, $\mathrm{S}_{\mathrm{m}}$, at design temperatures less than $500 \mathrm{~K}$ shall be the lower of:

- $\quad 2 / 3 \mathrm{~S}_{\mathrm{y}}$ (yield)

- $\quad 1 / 2 \mathrm{~S}_{\mathrm{u}}$ (ultimate)

as long as the reduction of area at fracture is greater than $40 \%$. For materials where this is not satisfied, a lower allowable stress limit should be considered in view of possible brittle behavior. A suggested guideline is a more conservative value of $\mathrm{S}_{\mathrm{m}}$ set at $1 / 2 \mathrm{~S}_{\mathrm{y}}$. Based on elastic analysis, the stress limits are:

- $\quad 1.0 \mathrm{KS}_{\mathrm{m}}$ for primary membrane stresses

- $\quad 1.3 \mathrm{KS}_{\mathrm{m}}$ for primary membrane plus bending stresses

- $\quad 1.5 \mathrm{KS}_{\mathrm{m}}$ for primary plus secondary stresses

For normal operating conditions, $\mathrm{K}=1.0$.

Clearly, at least in the outer half of the $\mathrm{CP}$ (at a radius greater than $0.5 \mathrm{~m}$ ), the material will be embrittled within 3 FPY. For primary membrane stresses under normal operating conditions, the allowable stress would be $1 / 2 \mathrm{~S}_{\mathrm{y}}$. High strength, high conductivity copper alloys tend to radiation harden in this temperature range. For conservatism, we assumed unirradiated strength properties. A wide range of strength properties is quoted in the literature for $\mathrm{CuCrZr}$, depending on the thermo-mechanical history of the material. For calculating the allowable stress in the CP, we assumed a yield strength of $350 \mathrm{MPa}$, which is consistent with the maximum yield strength that might be expected following a welding or brazing operation [6]. The allowable primary membrane stress would be $175 \mathrm{MPa}$. The peak VonMises stress (which approximates the Tresca stress) in the first option is $76 \mathrm{MPa}$ or $43 \%$ of the allowable primary membrane stress. The peak VonMises stress in the second option is $44 \mathrm{MPa}$ or $25 \%$ of the allowable primary membrane stress. Thus, it does not appear that primary stresses will be limiting.

Based on the ITER criteria, no stress (primary plus secondary) can exceed $1.5 \mathrm{KS}_{\mathrm{m}}$. For normal operation, the allowable stress for this combination of stresses in the CP would be $3 / 4 \mathrm{~S}_{\mathrm{y}}$ or $262 \mathrm{MPa}$. Stress concentrations around cooling holes in the range of 2-3 can be expected. This would result in a hoop stress of $120-180 \mathrm{MPa}$ (compression) around the cooling holes in the center of the $\mathrm{CP}$, rather than the average value of $60 \mathrm{MPa}$ (compression). Thermal stresses can also be expected due to temperature gradients around the cooling holes. The magnitude of these stresses is estimated to range from $33 \mathrm{MPa}$ (tension) around the cooling holes to $15 \mathrm{MPa}$ (compression) away from the cooling holes. Note that the thermal stress is opposite in sign to the hoop stresses that peak up around the cooling holes, thereby offsetting their impact. More detailed design and analysis and better material property data for laser formed conductor are required to make definitive judgements about the acceptability of the stresses for the TF coil design. However, these increments appear small relative to the margin between the allowable stress of $262 \mathrm{MPa}$ and the calculated primary membrane stress values of $76 \mathrm{MPa}$ and $43 \mathrm{MPa}$ for the first and second options respectively. Therefore, it appears promising that adequate structural margin exists for the ARIES-ST TF coil system design.

\section{CONCLUSION}

The TF coil system design addresses a number of the concerns (complexity) and criticisms (high cost, high recirculating power) of fusion. It does this by:

- Applying advanced, but available laser forming and spray casting techniques for manufacturing the TF coil system;

- Adopting a simplified single toroidal field coil system to make assembly and maintenance much easier. The single turn design also avoids the necessity of using the insulation as a structural component of the TF coils, and hence is much more robust than multi-turn designs.

- Using high conductivity copper and modest current densities to keep the recirculating power modest.

\section{ACKNOWLEDGMENTS}

This work was supported by the US DOE under Contract No. DE-AC02-76-CH03073.

\section{REFERENCES}

[1] F. Puhn et al, "Mechanical Design of a High-Beta TFCX Tokamak with a Demountable TF Coil," presented at the Thirteenth Symposium on Fusion Technology, Varese, Italy, September 1984.

[2] Holt Murray, "High Current Density, Cryogenically Cooled Sliding Electrical Joint Development”, PPPL2370, September, 1986

[3] F.A. Fabritsiev, S.J. Zinkle, and B.N. Singh, "Evaluation of copper alloys for fusion reactor divertor and first wall components", J. Nucl. Mater. (1996) Preprint

[4] Fusion Materials Semiannual Progress Report for the Period Ending December 1998, DOE/ER-0313/24: M.C. Billone, "Materials Input for the ARIES-ST Design Program," to be published

[5] ITER Design Criteria, June 1, 1995

[6] S.J. Zinkle and S.A. Fabritsiev, "Final Report to US Home Team for 1994 ITER R\&D Task T7: Assessment of Copper Alloys for High Heat Flux Applications" 